\title{
Combining doxorubicin with a phenolic extract from flaxseed oil: Evaluation of the effect on two breast cancer cell lines
}

\author{
ELIANA GUERRIERO ${ }^{1}$, ANGELA SORICE ${ }^{*}{ }^{*}$, FRANCESCA CAPONE $^{1 *}$, GABRIELLA STORTI $^{2}$, \\ GIOVANNI COLONNA ${ }^{3}$, GENNARO CILIBERTO ${ }^{4}$ and SUSAN COSTANTINI ${ }^{1}$
}

\author{
${ }^{1}$ CROM, Istituto Nazionale Tumori 'Fondazione G. Pascale' - IRCCS, Naples; ${ }^{2}$ Onco-Hematology Department, \\ Avellino Hospital, Avellino; ${ }^{3}$ Medical Informatics Service, University Hospital, Second University of Naples, \\ Naples; ${ }^{4}$ Scientific Direction, Istituto Nazionale Tumori 'Fondazione G. Pascale' - IRCCS, Naples, Italy
}

Received June 6, 2016; Accepted July 29, 2016

DOI: $10.3892 /$ ijo.2017.3835

\begin{abstract}
Breast cancer is one of the most frequently diagnosed forms of cancer and different treatments are used to block its progression. However, it still represents a very common cause of death in women. Doxorubicin (Dox) is reported as an effective agent in breast cancer treatment nonetheless it induces many side-effects. For this reason, many laboratories are engaged in understanding how it is possible to decrease the drug concentration, considering that one of the possible solutions is to use drug synergy, combining it with natural substances. Recently we showed that a phenolic extract from flaxseed (FS) oil, named PEFSO, induced on MCF-7 cell line an increase of apoptosis with related modification of G0/G1 phase cell cycle, and the activation of signaling and pro-oxidant pathways. In this study we present data on the combined effect of Dox and PEFSO on two different breast cancer cell lines to define the conditions to use lower doses of this chemotherapeutic agent. We report the data relating to the ability of this mixture to induce cytotoxicity and apoptosis, cell cycle modification, mitochondrial membrane depolarization and activation of extrinsic and/or intrinsic apoptotic pathway.
\end{abstract}

\section{Introduction}

Breast cancer is fatal among women (522.000 deaths in 2012) and one of the most frequently diagnosed cancers in 140 of 184 countries worldwide. Its clinical classification is based on the assessment of the type of expression of estrogen receptors (ERs), progesterone receptors (PRs), or human epidermal

Correspondence to: Dr Susan Costantini, CROM, Istituto Nazionale Tumori 'Fondazione G. Pascale', via Ammiraglio Bianco, 83013 Mercogliano, Avellino, Italy

E-mail: s.costantini@istitutotumori.na.it

*Contributed equally

Key words: doxorubicin, phenolic extract, breast cancer, apoptosis, cell cycle growth factor receptor 2 (HER2) (1). Triple-negative breast cancer (TNBC), which represents about $15 \%$ of cases, does not express any of these receptors, and, thus, it is more difficult to treat with current therapies, also because it is more likely to metastasize determining a poorer prognosis (1). Chemotherapy is routinely used for cancer treatment although its success is quite limited, due to severe side-effects caused by drug resistance, targeting of healthy cells, and metabolic stress (2). Doxorubicin (Dox) is one of the most effective agents in the treatment of breast cancer patients (3), even if it shows severe side-effects in the form of typhlitis, cardiotoxicity, nephrotoxicity, hepatotoxicity and other toxicities $(4,5)$. In general, Dox increases the oxidative stress, which kills cancer cells and induces an inflammatory microenvironment, with a generalized unwanted cellular toxicity (6); thus, Dox alone is not a preferable drug. A myriad of plant products have shown very promising anticancer properties in vitro, but they have yet to be evaluated in humans (7). However, some phytochemicals have shown a chemopreventive effect and the ability to sensitize cancer cells toward Dox (8). For example, recently it has been demonstrated that the combination of resveratrol and Dox in breast cancer is able to synergize their effects and to induce apoptosis, by downregulation of inflammatory factors, NF- $\mathrm{BB}, \mathrm{COX}-2$, and autophagy (LC3B, Beclin-1) (5). Even, ferulic acid showed a protective effect against cardiotoxicity induced by Dox in tumor-bearing mice as assessed by the level reduction of serum marker enzymes like CK and LDH and transaminases and activating lipid peroxidation (9). Furthermore, Dox-antioxidant compound, obtained by mixing Dox with ferulic acid, was found to be less toxic than Dox in cardiomyocytes (10). In the last two decades, flaxseed (FS) has been a focal point of interest in the field of nutrition and disease research due to the potential health benefits associated with its biologically active components (11). It has been shown that FS and/or its oil inhibit the formation of colon, breast, skin, and lung tumors reduce blood vessel cell formation in female rats, thus suggesting a protective effect against breast, colon and ovarian cancer (12). Since no detailed information was reported on the effect of the phenols in FS oil on breast cancer, we have characterized and assessed its potential effects on two human breast cancer cell lines, MCF-7 and MDA-MB231. Our results highlight 
that the main components of the phenolic extract from FS oil, named PEFSO, were ferulic, vanillic and p-hydroxybenzoic acids. They were very effective only on MCF-7 cell line by inducing an increase of apoptosis and lipid peroxidation, cell cycle $\mathrm{G} 0 / \mathrm{G} 1$ phase modification, and a related activation of the H2AX signaling pathway and of some pro-oxidant genes, when compared to untreated cells. The aim of the present study is to verify that PEFSO has a possible synergic effect with Dox. Therefore, we have analyzed the effects of their combination on ER-positive MCF-7 and receptor-negative MDA-MB231 human breast cancer cell lines in terms of cytotoxicity, apoptosis induction, cell cycle modification, mitochondrial membrane depolarization and gene expression involved in extrinsic/death receptor and mitochondrial/intrinsic apoptotic pathway.

\section{Materials and methods}

Cell culture and treatment. Two human breast cancer cell lines, estrogen receptor-positive MCF-7 (HTB-22, adenocarcinoma) and estrogen receptor-negative MDA-MB231 (HTB-26, adenocarcinoma) (both from Lonza, Verviers, Belgium) were grown as described elsewhere (13). Subsequently the cells were treated with Dox (Ebewe Pharma, Unterach, Austria) and PEFSO for $48 \mathrm{~h}$. For the MDA-MB231 cells, both compounds were dissolved in RPMI-1640 supplemented with $1 \% \mathrm{FBS}$ at concentrations of $0.09,0.18,0.38,0.75,1.5,3$, and $6 \mu \mathrm{M}$ for Dox and 4.03, 8.06, 16.13, 32.25, 64.5, 129, and $258 \mu \mathrm{g} / \mathrm{ml}$ for PEFSO. For MCF-7 cells, two compounds were dissolved in DMEM supplemented with $1 \%$ FBS at concentrations of 0.08 , $0.15,0.3,0.6,1.2,2.4$, and $4.8 \mu \mathrm{M}$ for Dox and 3.93, 7.88, 15.75, $31.5,63,126$, and $252 \mu \mathrm{g} / \mathrm{ml}$ for PEFSO. The concentrations used are similar to those of our previous studies $(13,14)$. The phenolic extract from FS oil was dissolved in dimethyl sulfoxide (DMSO; Sigma-Aldrich) at a concentration of $100 \mathrm{mM}$. In cell cultures the DMSO concentration remained always $<0.1 \%$, a dose that did not exert toxic effects (15). Subsequently according to the results, combination treatment (co-treatment) with Dox and PEFSO was carried out at $\mathrm{IC}_{50}$ doses obtained for $48 \mathrm{~h}$.

Sulforhodamine B (SRB) assay. The cell survival/proliferation was measured in 96-well plates by a spectrophotometric dye incorporation assay using SRB after $48 \mathrm{~h}$ of treatment with PEFSO, Dox and PEFSO + Dox. Cells were fixed with trichloroacetic acid (Sigma-Aldrich, St. Louis, MO, USA) for $1 \mathrm{~h}$ and after stained for $30 \mathrm{~min}$ with $0.4 \%$ (w/v) SRB (Sigma-Aldrich) dissolved in $1 \%$ acetic acid. The number of viable cells was directly proportional to the protein bound-dye formation which was then solubilized with $10 \mathrm{mM}$ Tris base solution $\mathrm{pH} 10.5$ and measured by fluorometric assay ELISA at $540 \mathrm{~nm}$ (microplate reader; Bio-Rad, Hercules, CA, USA). All experiments were performed in duplicate and repeated three times. Cellular viability was estimated as \% compared to untreated cells. The $\mathrm{IC}_{50}$ was assessed from the dose-response curves.

Drug combination studies. Drug combination studies were based on concentration-effect curves generated as a plot of the fraction of unaffected (surviving) cells vs. the drug concentration after $48 \mathrm{~h}$ of treatment. Briefly, the two molecules were tested for $48 \mathrm{~h}$ in combination equiactive doses (cytotoxic ratio, 50:50). Synergism, additivity, and antagonism were quantified by determining the combination index (CI) calculated by the Chou-Talalay equation and with the software CalcuSyn (Biosoft, Cambridge, UK) (16). Assuming 0.9 as the cut-off value, $\mathrm{CI}<0.9$; $\mathrm{CI}, 0.9-1$; or $\mathrm{CI}>1$ indicates synergistic, additive, or antagonistic effects, respectively. The dose reduction index (DRI) represents the measure of how much the dose of each substance in a synergistic combination may be reduced at a given effect level compared with the dose of each drug alone. The linear correlation coefficient (r) of the median-effect plot is considered a conformity measure of the data according to the mass-action law principle when the experimental measurement is assumed to be accurate. An r-value equal to 1 indicates perfect conformity while a poor value may be the result of biological variability or experimental deviations. All our experiment r-values were between 0.91 and 0.98 indicating a good data conformity.

Apoptosis assay. The cells $\left(1 \times 10^{6}\right)$ were labeled with Annexin V and Dead Cell Assay kit according to the manufacturer's instructions (Merck Millipore, Darmstadt, Germany) after they were harvested and washed twice with ice-cold PBS. This test is based on the phosphatidylserine (PS) detection on the apoptotic cell surface, using fluorescently labeled Annexin $\mathrm{V}$ in combination with the dead cell marker, 7-aminoactinomycin D (7-AAD). The apoptotic ratio was calculated by identifying four populations: i) Annexin V (-) and dead cell marker (-), the viable cells; ii) Annexin V (+) and dead cell marker (-), the early apoptotic cells; iii) Annexin V (+) and dead cell marker (+), the late apoptotic cells; and iv) Annexin V (-) and dead cell marker $(+)$, the cells died through non-apoptotic pathway. The samples were counted and analyzed by the Muse $^{\mathrm{TM}}$ Cell Analyzer and a software provided by Merck Millipore, respectively.

Cell cycle analysis. Cell cycle analysis was performed utilizing Muse ${ }^{\mathrm{TM}}$ Cell Analyzer following the manufacturer's instructions. After treatments, cells were washed with PBS, centrifuged and after removal of the supernatant, $1 \mathrm{ml}$ of ice-cold $70 \%$ ethanol was added to the cell pellet. The samples were capped and frozen at $-20^{\circ} \mathrm{C}$ for at least $3 \mathrm{~h}$ prior to staining. Ethanol-fixed cells were centrifuged and the pellet was re-suspended in PBS. After a further centrifugation, the supernatant was removed and discarded and cell pellet was re-suspended in $200 \mu \mathrm{l}$ of Muse ${ }^{\mathrm{TM}}$ Cell Cycle Reagent containing propidium iodide (PI) and RNase A in a proprietary formulation. PI discriminates cells at different stages of the cell cycle, based on the differential DNA content in the presence of RNase to increase the specificity of DNA staining. The cells were incubated for $30 \mathrm{~min}$ at room temperature, in the dark. After staining, the cells were processed for cell cycle analysis.

RNA preparation and quantitative reverse transcription polymerase chain reaction $(R T-q P C R)$. RNA isolation and cDNA preparation were performed as previously described by Sorice et al (13). The reverse transcribed products were used to perform qPCR in order to evaluate the expression level of transcripts of selected genes. Sequences for mRNAs from 
the nucleotide data bank (National Center for Biotechnology Information, Bethesda, MD, USA) were used to design primer pairs for RT-qPCR (Primer Express Software; Applied Biosystems, Foster City, CA, USA). Oligonucleotides were obtained from Sigma-Aldrich. The efficiency of each primer pair was calculated according to the standard curve method using the equation $\mathrm{E}=10^{-1 / \mathrm{slope}}$. Five serial dilutions were set up to determine $\mathrm{Ct}$ values and reaction efficiencies for all primer pairs. Standard curves were generated for each oligonucleotide pair using $\mathrm{Ct}$ values vs. the logarithm of each dilution factor. RT-qPCR assays were run on the 7900HT Fast Real-Time PCR System (Applied Biosystems).

The primer sequences are provided in Table I. Starting with $2 \mu \mathrm{g}$ of total RNA, we have prepared a 20 -fold dilution of the resulting cDNA to achieve the concentration equivalent of starting with $100 \mathrm{ng}$ of RNA (Life Technologies/Invitrogen), according to the manufacturer's instructions. A total of $10 \mathrm{ng}$ of cDNA was amplified in a total volume of $25 \mu$ l containing 1X SYBR-Green PCR Master Mix (Applied Biosystems) and $300 \mathrm{nM}$ of forward and reverse primers. The thermal profile conditions were as follows: $5 \mathrm{~min}$ of denaturation at $95^{\circ} \mathrm{C}$ followed by 44 cycles at $95^{\circ} \mathrm{C}$ for $30 \mathrm{sec}$ and $60^{\circ} \mathrm{C}$ for $1 \mathrm{~min}$. We have added one cycle for melting curve analysis at $95^{\circ} \mathrm{C}$ for $15 \mathrm{sec}, 60^{\circ} \mathrm{C}$ for $15 \mathrm{sec}$ and $95^{\circ} \mathrm{C}$ for $15 \mathrm{sec}$ to verify the presence of a single product. Melting curve analysis was carried out after amplification to verify the validity of the amplicon. Each assay included a no-template control for each primer pair. To capture intra-assay variability, all RT-qPCR reactions were carried out in triplicate. For all RT-qPCR experiments, the data from each cDNA sample were normalized using $\beta$-actin mRNA as endogenous level (17). Sample $\Delta \mathrm{Ct}$ values were calculated as the difference between the means of gene markers $\mathrm{Ct}$ and housekeeping assay $\mathrm{Ct}$ from the same sample. The 1-fold expression level was chosen as the threshold for significance of target genes. Statistical analyses (paired Student's t-tests) were performed using Prism software (GraphPad Software, Inc., La Jolla, CA, USA).

Mitochondrial membrane depolarization. Measurement of changes in the mitochondrial membrane potential $(\Delta \Psi \mathrm{m})$ was performed with the Muse MitoPotential Assay kit ${ }^{\mathrm{TM}}$ (EMD Millipore). The assay utilizes the MitoPotential Dye, a cationic, lipophilic dye to detect changes in the $\Delta \Psi \mathrm{m}$ and 7-AAD as an indicator of cell death. High membrane potential drives the accumulation of MitoPotential Dye within inner membrane of intact mitochondria resulting in high fluorescence, while cells with depolarized mitochondria demonstrate a decrease in fluorescence. Therefore, this flow cytometry-based assay differentiates four populations of cells: live cells with depolarized mitochondrial membrane, MitoPotential//7-AAD; live cells with intact mitochondrial membrane, MitoPotential ${ }^{+} / 7-\mathrm{AAD}^{-}$; dead cells with depolarized mitochondrial membrane, MitoPotential ${ }^{+} / 7-\mathrm{AAD}^{+}$; and dead cells with intact mitochondrial membrane, MitoPotential $/ 7$-AAD ${ }^{+}$. After treated with different concentrations of drugs, the cells were incubated with the fluorescent dyes and the percentage of depolarized cells (depolarized live + depolarized dead) were determined by Muse Cell Analyzer. We measured with Muse MitoPotential Assay two important cell health parameters: change in mitochondrial
Table I. Primer sequences of the genes used in this study.

\begin{tabular}{ll}
\hline Gene name & \multicolumn{1}{c}{ Primer sequence $\left(5^{\prime} \rightarrow 3^{\prime}\right)$} \\
\hline p38 MAPK & GCC CAA GCC CTT GCA CAT (18) \\
& TGG TGG CAC AAA GCT GAT GAC (21) \\
p53 & CTG GCC CCT GTC ATC TTC TG (20) \\
& CCG TCA TGT GCT GTG ACT GC (20) \\
Bax & GGA CGA ACT GGA CAG TAA CAT GG (23) \\
Caspase-3 & GCA AAG TAG AAA AGG GCG ACA AC (23) \\
& TGG CAC AAA GCG ACT GGA T (19) \\
Caspase-8 & GGA TGG CCA CTG TGA ATA ACT G (22) \\
& TCG AGG ACA TCG CTC TCT CA (20) \\
$\beta$-actin & TCT GGC ACC ACA CCT TCT ACA ATG (24) \\
& AGC ACA GCC TGG ATA GCA ACG (21)
\end{tabular}

MAPK, mitogen-activated protein kinase. Numbers in parentheses denote the primer lengths.

potential and cell death. The software provides percentages of live, depolarized, depolarized/death and death cells. Briefly cells, after the treatment with PEFSO alone or in combination with Dox, were harvested and the cell pellet was suspended in assay buffer. MitoPotential Dye working solution was added and the cell suspension incubated at $37^{\circ} \mathrm{C}$ for $20 \mathrm{~min}$. After the addition of Muse MitoPotential 7-AAD dye and incubation for $5 \mathrm{~min}$, changes in $\Delta \Psi \mathrm{m}$ and in cellular plasma membrane permeability were assessed using the fluorescence intensities of both analyzed dyes by Muse Cell Analyzer, flow cytometry.

\section{Results}

Cytotoxicity assay. The cytotoxic effects of Dox and its combination with PEFSO were evaluated on MCF-7 and MDA-MB231 cell lines by SRB assay to identify the concentrations at which the $50 \%$ of cell growth was inhibited. As reported in our recent report (13), after the treatment with PEFSO alone the MCF-7 and MDA-MB231 cells reached an inhibition corresponding to an $\mathrm{IC}_{50}$ of 63 and $64.5 \mu \mathrm{g} / \mathrm{ml}$, respectively. In the case of Dox treatment the two cell lines reached their respective $\mathrm{IC}_{50}$ values at concentrations of 1.2 and $1.5 \mu \mathrm{M}$ for MCF-7 and MDA-MB231 cells, respectively, when compared to non-treated cells (Fig. 1). Subsequently, on the basis of the median value obtained from the effect analysis of Dox and PEFSO alone in calculating CIs, we explored the anti-proliferative effects of Dox and PEFSO combinations by testing equipotent doses of the two agents (ratio, 50:50). In this way we have verified that the MCF-7 and MDA-MB231 cells reached an $\mathrm{IC}_{50}$ inhibition comparable to those of stimulations with $15.75 \mu \mathrm{g} / \mathrm{ml}$ (PEFSO) and $0.3 \mu \mathrm{M}$ (Dox) and with $24 \mu \mathrm{g} / \mathrm{ml}$ (PEFSO) and $0.49 \mu \mathrm{M}$ (Dox), respectively. A strong synergistic effect with low CIs $(\mathrm{CIs}<0.9)$ was demonstrated when simultaneous equipotent combination doses were used for both cell lines (Fig. 1). Therefore, after combined treatment we have achieved a dose reduction of 19.46-fold for Dox and 7.74-fold for PEFSO in MCF-7 cells at $\mathrm{IC}_{50}$ values (DRI50) as well as of 3.10-fold and 4.62-fold for Dox and PEFSO in 
Table II. PEFSO and Dox co-treatment induced a synergistic anti-proliferative effect compared to the treatment with drugs administered individually as demonstrated by median drug effect analysis calculating the CI and the DRI with CalcuSyn software.

\begin{tabular}{lccrrr}
\hline & & & & DRI at IC $50( \pm$ SD $)$ \\
\cline { 3 - 6 } Cell lines & Treatment & CI50 $( \pm$ SD $)$ & $r( \pm$ SD $)$ & Dox & PEFSO \\
\hline MCF-7 & PEFSO + Dox & $0.2( \pm 0.01)$ & $0.94( \pm 0.03)$ & $19.46( \pm 0.05)$ & $7.74( \pm 0.06)$ \\
MDA-MB231 & PEFSO + Dox & $0.5( \pm 0.01)$ & $0.97( \pm 0.02)$ & $3.10( \pm 0.08)$ & $4.62( \pm 0.05)$ \\
\hline
\end{tabular}

Dox, doxorubicin; co-treatment, combination treatment; CI, combination index; DRI, dose reduction index.

MCF-7
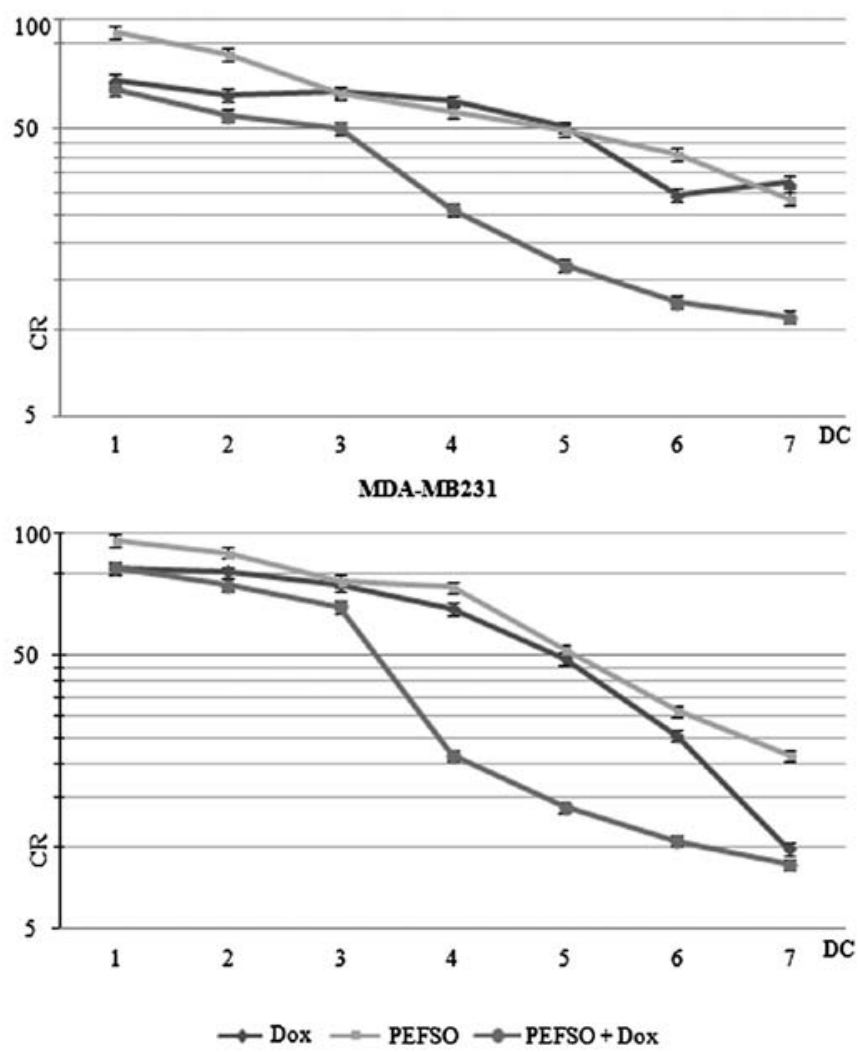

Figure 1. SRB assays. MCF-7 and MDA-MB231 CR curves after treatment with Dox, PEFSO and their combination for $48 \mathrm{~h}$. On the $\mathrm{x}$-axis the different DCs are shown: 1 (Dox: $0.08 \mu \mathrm{M}$; PEFSO: $3.93 \mu \mathrm{g} / \mathrm{ml}$ ), 2 (Dox: $0.15 \mu \mathrm{M}$; PEFSO: $7.88 \mu \mathrm{g} / \mathrm{ml}$ ), 3 (Dox: $0.3 \mu \mathrm{M}$; PEFSO: $15.75 \mu \mathrm{g} / \mathrm{ml}$ ), 4 (Dox: $0.6 \mu \mathrm{M}$; PEFSO: $31.5 \mu \mathrm{g} / \mathrm{ml}$ ), 5 (Dox: $1.2 \mu \mathrm{M}$; PEFSO: $63 \mu \mathrm{g} / \mathrm{ml}$ ), 6 (Dox: $2.4 \mu \mathrm{M}$; PEFSO: $126 \mu \mathrm{g} / \mathrm{ml}$ ) and 7 (Dox: $4.8 \mu \mathrm{M}$; PEFSO: $252 \mu \mathrm{g} / \mathrm{ml}$ ) for MCF-7 cells; and 1 (Dox: $0.09 \mu \mathrm{M}$; PEFSO: $4.03 \mu \mathrm{g} / \mathrm{ml}$ ), 2 (Dox: $0.18 \mu \mathrm{M}$; PEFSO: $8.06 \mu \mathrm{g} / \mathrm{ml}$ ), 3 (Dox: $0.38 \mu \mathrm{M}$; PEFSO: $16.13 \mu \mathrm{g} / \mathrm{ml}$ ), 4 (Dox: $0.75 \mu \mathrm{M}$; PEFSO: $32.25 \mu \mathrm{g} / \mathrm{ml}$ ), 5 (Dox: $1.5 \mu \mathrm{M}$; PEFSO: $64.5 \mu \mathrm{g} / \mathrm{ml}$ ), 6 (Dox: $3 \mu \mathrm{M}$; PEFSO: $129 \mu \mathrm{g} / \mathrm{ml}$ ) and 7 (Dox: $6 \mu \mathrm{M}$; PEFSO: $258 \mu \mathrm{g} / \mathrm{ml}$ ) for MDA-MB231 cells. On the y-axis: CR. SRB, sulforhodamine; CR, cell growth rate; Dox, doxorubicin; DCs, drug concentrations.

MDA-MB231 cells, respectively, when compared to concentrations of two compounds taken individually (Table II).

Apoptosis studies. Our previous data showed that the treatment with 63 and $64.5 \mu \mathrm{g} / \mathrm{ml}$ of PEFSO alone in MCF-7 and MDA-MB231 cells resulted in induction of apoptotic death equal to $82.05 \%( \pm 0.02)$ and $22.95 \%( \pm 0.04)$, respectively (13). Therefore, we investigated also the Dox ability
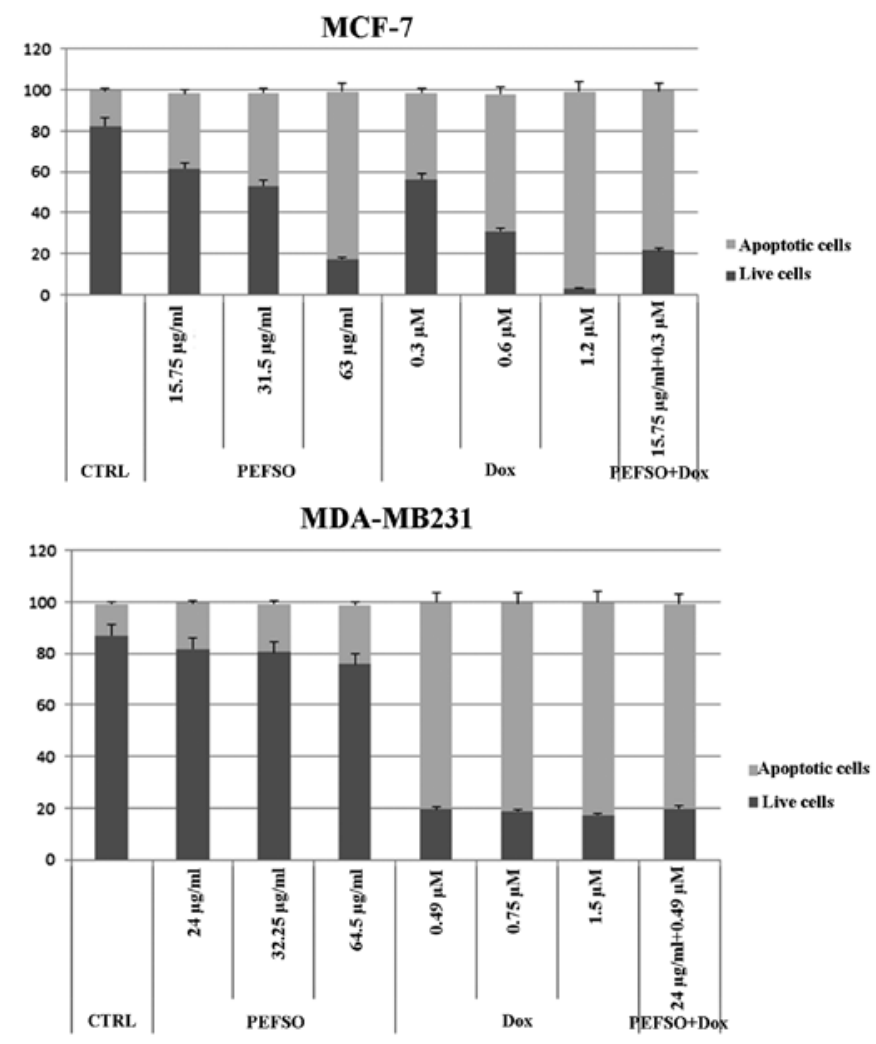

Figure 2. Percentage of live and apoptotic cells by the Muse Annexin $\mathrm{V}$ and Dead Cell Assay in (A) MCF-7 and in (B) MDA-MB231 cells after PEFSO, Dox and co-treatment at $48 \mathrm{~h}$. Dox, doxorubicin; co-treatment, combination treatment.

and its combination with PEFSO to induce apoptosis in the two cell lines. Fig. 2 shows that the treatments with only Dox at $\mathrm{IC}_{50}$ concentrations induced an apoptotic death equal to $96.36 \%( \pm 0.08)$ and $82.08 \%( \pm 0.06)$, in $\mathrm{MCF}-7$ and MDA-MB231 cells, respectively. The treatment with $\mathrm{PEFSO}+$ Dox concentrations corresponding at $\mathrm{IC}_{50}$, like $0.3 \mu \mathrm{M}$ (Dox) $+15.75 \mu \mathrm{g} / \mathrm{ml}$ (PEFSO) for MCF-7 cells and $0.49 \mu \mathrm{M}$ (Dox) $+24 \mu \mathrm{g} / \mathrm{ml}$ (PEFSO) for MDA-MB231 cells, resulted in induction of apoptotic death equal to $77.90 \%$ $( \pm 0.01)$ and $79.31 \%( \pm 0.05)$, in MCF-7 and MDA-MB231 cells, respectively. This confirmed the specific synergistic effect of this combination by evidencing that the advantage, which is achieved with a combined formulation, is due to the fact that the dose of the chemotherapeutic agent is significantly reduced, from 1.2 to $0.3 \mu \mathrm{M}$ for MCF-7 cells and from 1.5 to $0.49 \mu \mathrm{M}$ for MDA-MB231 cells. 
MCF-7

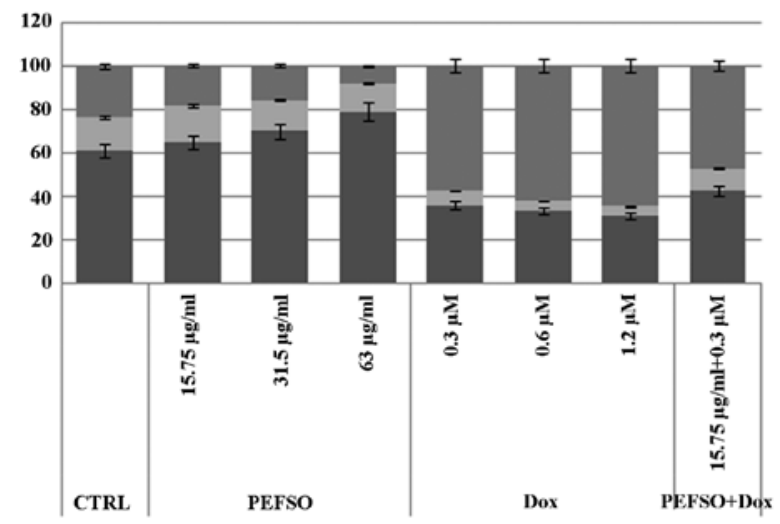

MDA-MB231

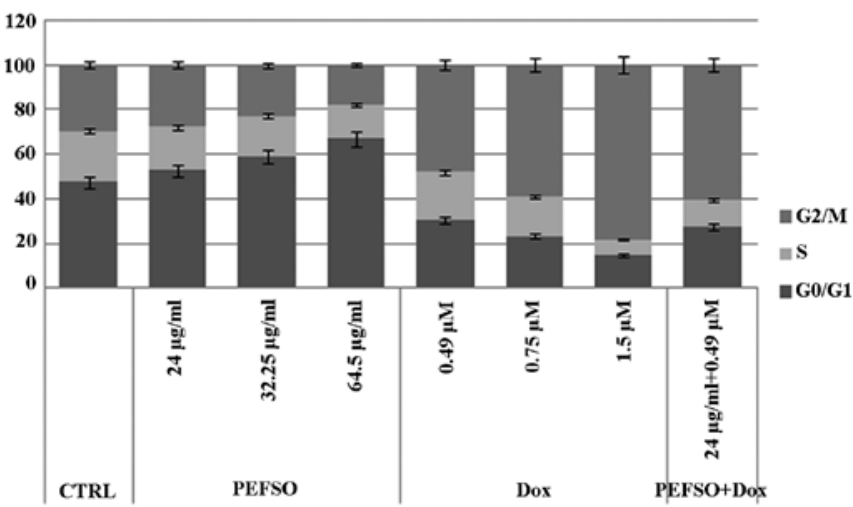

Figure 3. Cell percentages in G0/G1, S and G2/M phases in MCF-7 and in MDA-MB231 cells upon PEFSO, Dox and co-treatment at 48 h. Dox, doxorubicin; co-treatment, combination treatment.

Cell cycle assay. Considering that the two compounds affected the cell proliferation inducing death in both cell lines, we analyzed their effects also on the cell cycle distribution after $48 \mathrm{~h}$ of treatment at the same concentrations used for the apoptosis assay. In our recent report, we observed in both cell lines a dose-dependent increase of the percentage of cells in G0/G1 phase as well as a decrease in G2/M and a slight decrease in S phase, when treated with PEFSO and compared to the control (13). After treatment by Dox, we have a decrease of G0/G1 and S phases and an increase of G2/M phase in both cell lines (Fig. 3). While after treatment with PEFSO + Dox at concentrations corresponding to $\mathrm{IC}_{50}$, we had an increase of $\mathrm{G} 2 / \mathrm{M}$ phase similar to that of Dox alone. However, even if the data after the co-treatment are not comparable to those obtained using Dox and PEFSO alone, it is important to underline that the concentrations used during the co-treatment were lower than $\mathrm{IC}_{50}$ values obtained by dose-response assays with Dox and PEFSO alone, and that the effects after Dox + PEFSO are certainly influenced from two different action mechanisms due to the two molecules.

Mitochondrial membrane depolarization. We evaluated, on both cell lines, the effects on depolarization of the mitochondrial membranes (loss of $\Delta \Psi \mathrm{m}$ ) by Muse system when treated with the two compounds alone or in combination. Loss of the mitochondrial inner transmembrane potential is a reliable indicator of mitochondrial dysfunction and cellular health. This effect is often observed to be associated with the early

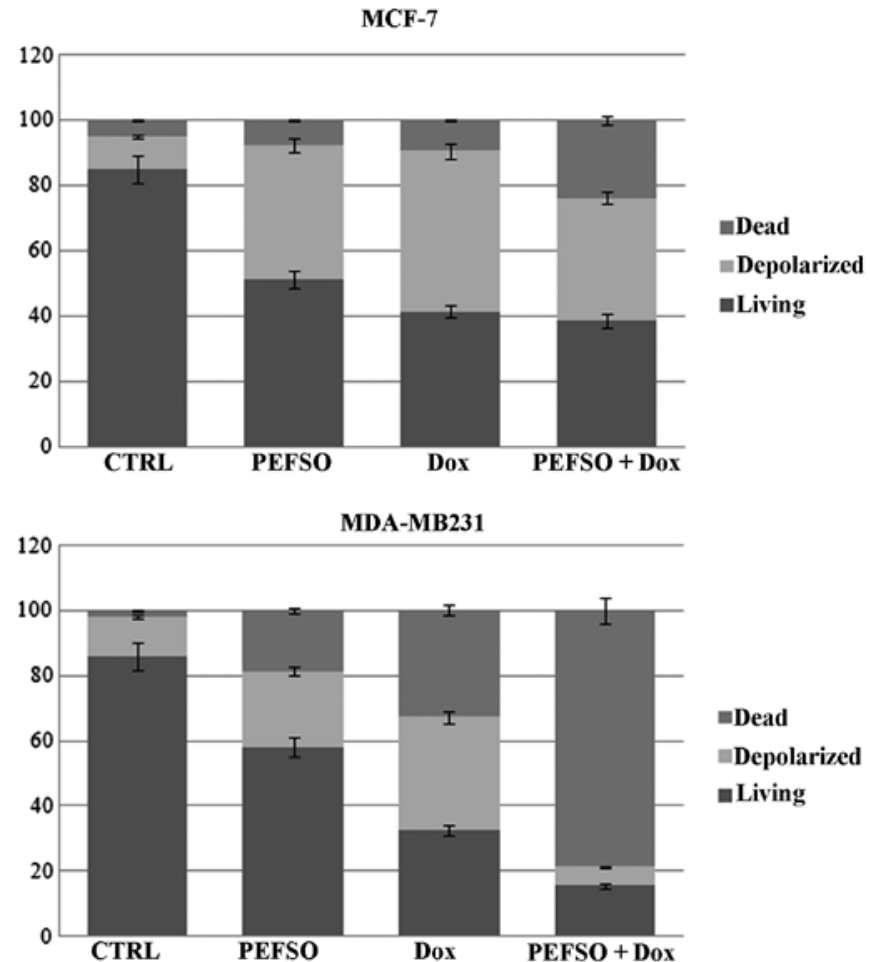

Figure 4. The histograms show $\Delta \Psi \mathrm{m}$ after PEFSO treatment $(63 \mu \mathrm{g} / \mathrm{ml}$ on MCF-7 and $64.5 \mu \mathrm{g} / \mathrm{ml}$ on MDA-MB231 cells), after Dox treatment (1.2 $\mu \mathrm{M}$ on MCF-7 and $1.5 \mu \mathrm{M}$ on MDA-MB231 cells), and after their co-treatment $(15.75 \mu \mathrm{g} / \mathrm{ml}$ of PEFSO $+0.3 \mu \mathrm{M}$ of Dox on MCF-7 cells and $24 \mu \mathrm{g} / \mathrm{ml}$ of PEFSO and $0.49 \mu \mathrm{M}$ of Dox on MDA-MB231 cells). $\Delta \Psi \mathrm{m}$, mitochondria membrane potential; Dox, doxorubicin; co-treatment, combination treatment.

stages of apoptosis (18). We observed in MCF-7 cell line an increase of the mean percentage of cellular depolarization in presence of $\mathrm{IC}_{50}$ concentrations for PEFSO and Dox, respectively, when compared to untreated. Their combination at two concentrations below the $\mathrm{IC}_{50}$ values produced a nearly similar depolarization in respect to each compound alone. Furthermore, we noted an increase of death cells by means of co-treatment that we had not observed during the treatment with the individual compounds (Fig. 4). A significant change in the $\Delta \Psi \mathrm{m}$ was also evident in MDA-MB231 cells compared to the control. We observed also an increase of cell death when treated with PEFSO and Dox alone, compared to the control. Interestingly, cells co-treated with two concentrations below the $\mathrm{IC}_{50}$ values (15.75 and $24 \mu \mathrm{g} / \mathrm{ml}$ for PEFSO, 0.3 and $0.49 \mu \mathrm{M}$ for Dox in MCF-7 and MDA-MB231 cells, respectively) showed a decrease of depolarization and a significant increase of cell death (Fig. 4). This is evidence that the co-treatment is able to activate two different death pathways in the two cancer cell lines.

$R T$ - $q P C R$ analysis. To further elucidate the molecular mechanism through which PEFSO and Dox and their combination were able to induce apoptosis in breast cancer cells, we have examined mRNA expression of certain genes involved in the intrinsic mitochondrial pathway such as p53, Bax, p38, and caspase-3 as well the extrinsic death receptor pathway such as those of caspase- 3 and -8 , by focusing mainly on the activation mechanism of caspase-3 (Table I). RT-qPCR was used to detect the mRNA expression after separate treatment with Dox 
or PEFSO at their $\mathrm{IC}_{50}$ concentrations as well as at two lower $\mathrm{IC}_{50}$ concentrations combined. mRNA expression change was normalized on $\beta$-actin mRNA expression (Fig. 5). Results showed that the mRNA expression of p53, Bax, p38, and caspase-3 genes increased significantly after treatment for $48 \mathrm{~h}$ with Dox, PEFSO and their combination in MCF-7 cell line. On the contrary, no increase of caspase- 8 gene expression was highlighted in this cell line after treatment with Dox and PEFSO taken individually, while an increase of its expression is noticed when the two compounds are combined. Caspase- 8 is involved in an extrinsic apoptotic pathway activated by a death receptor. Taken together, these observations indicated that Dox and PEFSO induced only the intrinsic apoptotic pathway while their combination induces apoptosis by both intrinsic and extrinsic pathways. In MDA-MB231 cell line the two compounds, PEFSO and Dox, caused a significant increase of p53, Bax, caspase-3, p38 and caspase- 8 expression indicating an activation of both apoptotic pathways. Their combination did not show any significant increase of p53, Bax and p38 expression levels, while caspase- 3 and -8 expression levels were activated. Therefore, we supposed that Dox and PEFSO combination in MDA-MB231 cells induced only activation of genes involved in the extrinsic apoptotic pathway, whereas when used individually they activate both pathways.

\section{Discussion}

Dox is an anthrax-cyclin antibiotic, which remains an important agent in many chemotherapy regimens (3). Although Dox is currently considered to be one of the most effective agents in the treatment of human breast cancer, its chemotherapeutic use is associated with severe side-effects to non-tumor tissues, such as the heart, liver, and kidney, thus greatly limiting its clinical application (19). In recent years, FS have attracted considerable interest for their potential health benefits, including the prevention of chronic non-communicable diseases, the cardiovascular disease reduction, atherosclerosis, diabetes, cancer, arthritis, osteoporosis, and neurological disorders $(11,20,21)$. Different studies have also reported that the FS components are effective in reducing breast cancer risk and tumor growth, or in interacting beneficially with breast cancer drugs (22). In particular, in our recent study we also characterized the phenolic components extracted from FS oil (PEFSO) and analyzed their anticancer effect on two human breast cancer cell lines, MCF-7 and MDA-MB231, and on the human non-cancerous breast cell line, MCF-10A (13). Therefore, in this study we investigated the effect of the combination of PEFSO with Dox in order to define its ability to reduce the doses of this chemotherapeutic agent also decreasing its side-effects.

Hence considering that in our study (13) the healthy breast cells, MCF-10A, retained a quite constant viability with increasing concentrations of PEFSO and that, hence, this extract was not able to induce modulation of apoptosis and cell cycle on MCF-10A, we decided to test the effects of the combination between Dox and PEFSO only on breast cancer cell lines.

Firstly we observed that Dox in combination with PEFSO had anti-proliferative effects reaching $\mathrm{IC}_{50}$ at concentrations
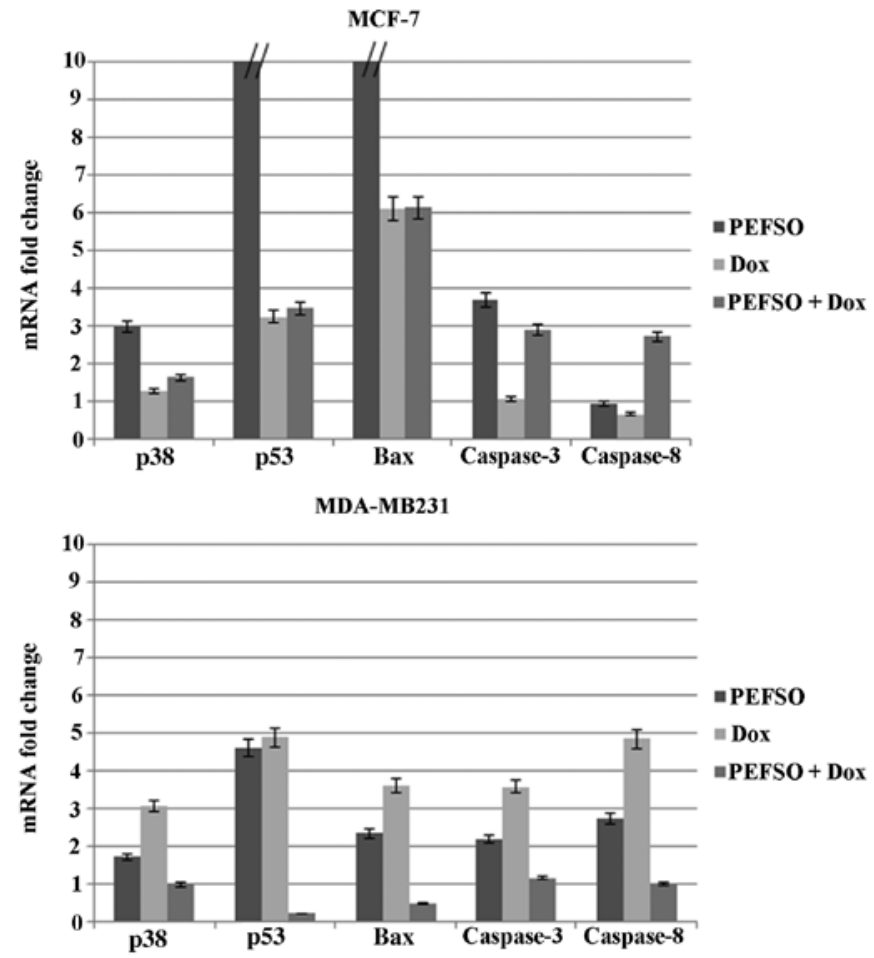

Figure 5. RT-qPCR analysis. The fold changes evaluated as ratio between the expression levels of five genes in the breast cancer MCF-7 and MDA-MB231 cells upon PEFSO, Dox and co-treatment for $48 \mathrm{~h}$ compared to those in the untreated cells. On y-axis mRNA fold changes. RT-qPCR quantitative reverse transcription polymerase chain reaction; Dox, doxorubicin; co-treatment, combination treatment.

equal to 0.3 and $0.49 \mu \mathrm{M}$ in MCF-7 and MDA-MB231 cell lines, respectively. These $\mathrm{IC}_{50}$ combination values are lower than the concentration of Dox alone, 1.2 and $1.5 \mu \mathrm{M}$ in MCF-7 and MDA-MB231, respectively, which is able to induce an anti-proliferative effect also in comparison with data already reported in literature where Dox concentrations (ranging from 0.1 to $10 \mu \mathrm{M})$ decreased the viability of MCF-7 cells in a time- and concentration-dependent manner (23). This supports our view that this natural compound in combination with Dox, a conventional breast cancer chemotherapeutic agent, is useful to enhance the drug antitumor activity reducing its active concentration and the adverse toxic effect. In particular, we found that the strongly synergistic combination of the two compounds in both cell lines, as evaluated by means of CalcuSyn software, induced an increase of apoptosis and a modulation of cell cycle through a decrease of G0/G1 and S phases and an increase of G2/M phase similarly to Dox, when individually used; in fact, Dox was able to arrest the MCF-7 and T47D breast cancer cell lines at G2/M phase (24). Different studies also have indicated that Dox-induced apoptosis is associated with two distinct apoptotic pathways, i.e., the extrinsic and mitochondrial or intrinsic pathways (19). The extrinsic pathway involved the death receptors and ligand interaction such as FasL/Fas and then activated caspase- 8 (25). It is reported that caspase- 8 levels increased in MCF-7 cell line after a 48- and 72-h incubation with 0.1 and $1 \mu \mathrm{M}$ concentrations of Dox (23). Moreover, upregulation of pro-caspase- 8 was found upon treatment with Dox in colon carcinomas cells (26). The mitochondrial or intrinsic pathway is the major mechanism of Dox-induced 
A
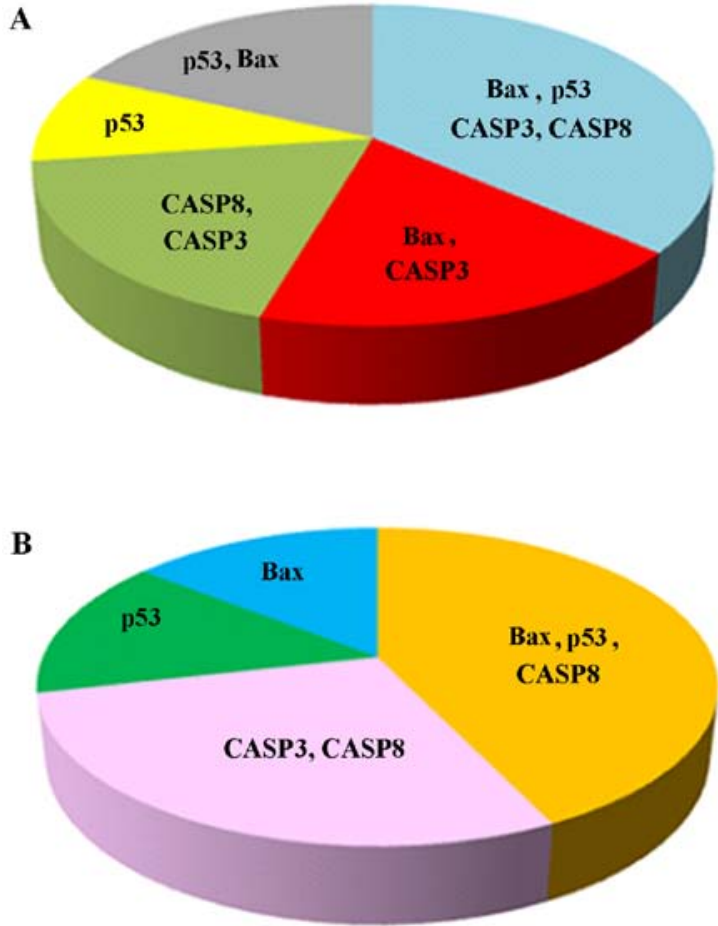

Apoptosis signaling pathway

mCCKR signaling map

= FAS signaling pathway

Wnt signaling pathway

"1053 pathway

Binding

Catalytic activity

Nucleic acid binding

transcription factor activity

mTransporter activity

Figure 6. (A) Pathway and (B) molecular function analysis by PANTHER program.

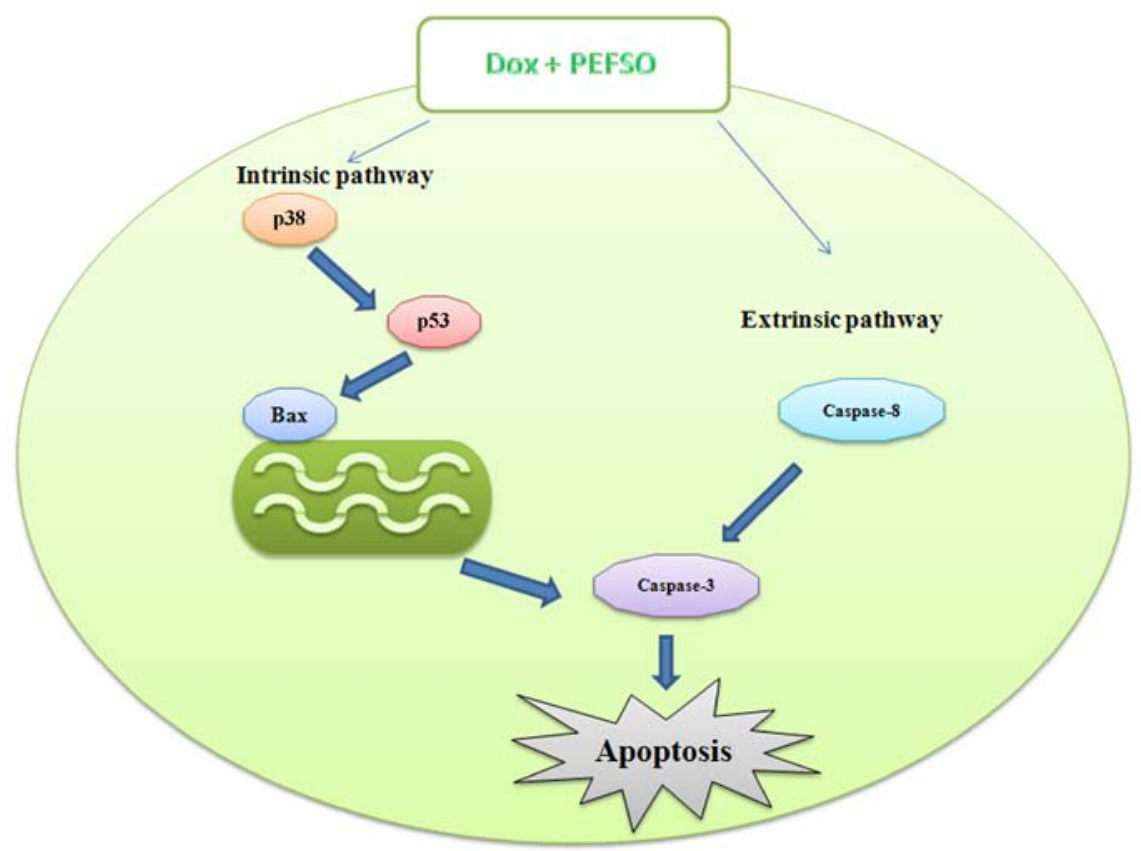

Figure 7. Scheme related to the activation of apoptotic intrinsic and extrinsic pathways from Dox + PEFSO. Dox, doxorubicin.

apoptosis, in which the central process involves the change of permeability of the outer mitochondrial membrane with the subsequent release of several pro-apoptotic factors into the cytosol (23). Furthermore, Dox causes apoptosis of bone marrow-derived mesenchymal stem cells (BMSCs) through ROS increase and the loss of $\Delta \Psi \mathrm{m}$, as well as the activation of p38, p53, Bax and caspase- 3 genes, which consequently trigger apoptosis and dysfunction of cells (27). According to data reported in literature, we decided to investigate certain apoptosis-associated genes, which might contribute to Dox-induced apoptosis and to its combination with PEFSO. Fig. 6 shows the functional and pathway analysis performed by PANTHER program (28) on the chosen genes showing their involvement also in other signaling pathways underlining their important role in cancer. First, we analyzed the p53 gene expression that can directly trigger the permeability of the outer mitochondrial membrane through activation of pro-apoptotic proteins such as Bax (26). High expression of 
Bax gene in MCF-7 cells incubated with Dox demonstrated that there are Bax enhancer effects followed by induction of the intrinsic apoptotic pathway (23). Indeed, we found activation of p53 and Bax in MCF-7 and MDA-MB231 cell lines treated with Dox and PEFSO alone. Their combination activates these genes only in MCF-7 cells, not in MDA-MB231 cells.

Moreover, a recent study has shown that the mitogen-activated protein kinase (MAPKs) signaling is able to regulate apoptosis-associated pathways in tumor cells $(29,30)$ where p38 protein is involved in intrinsic pathway and appears to have a pro-apoptotic effect activating a variety of cellular stress and dysfunctions of mithocondria and caspase activation in cell apoptosis (31). In our study Dox and PEFSO induce p38 MAPK increase in both cell lines but their combination has effect on p38 activation only in MCF-7 cells. However, regarding caspases it is important to underline that the cell death induction of both extrinsic and intrinsic apoptotic pathways is associated with caspase activation, where caspase-8, which can directly activate caspase-3 (32), is activated mainly in the extrinsic apoptotic pathway (23). Both pathways converged on caspase-3, and later on other enzymes lead to final events of apoptosis (33), for this reason our aim was mainly to evaluate its role in the activation of the intrinsic and extrinsic pathway of apoptosis and how it was activated.

Overall, in our study we found that: i) caspase-3 levels increased after a 48-h incubation with Dox and PEFSO alone and after their combination in both breast cell lines but; ii) caspase- 8 levels did not increase in MCF-7 cells after treatment with the two compounds, only when the cells were stimulated with Dox + PEFSO alone; and iii) in MDA-MB231 cells, caspase- 8 increased always in both individual treatment and in co-treatment. Hence, these data demonstrate that in MCF-7 cell line Dox + PEFSO induce an apoptotic intrinsic pathway by p53, Bax p38 and caspase-3 activation as well as an apoptotic extrinsic pathway by caspase- 8 activation. In MDA-MB231 cells, the cellular death may be mediated through both extrinsic and intrinsic apoptotic pathways when the compounds are used individually, but their combination activated only the extrinsic pathway (Fig. 7). These results have been confirmed by the assessment of $\Delta \Psi$ m by Muse system. Indeed, in the MDA-MB231 co-treatment we did not find any mitochondrial membrane depolarization but an increase of dead cells. This did not happen when the two compounds were used individually. However, several studies have also shown that in the extrinsic pathway, there is a caspase- 8 activation, which bypasses mitochondria and leads directly to caspase-3 activation, followed by apoptosis $(33,34)$. In MCF-7 cells we observed depolarization of the mitochondrial membrane when the compounds were used individually as well as when they were combined.

Therefore, on the basis of our results, the combined use of the natural product, PEFSO, with the conventional chemotherapy drug, Dox, could be proficiently used to decrease the Dox effective dose and, hence, most likely also its side-effects.

\section{Acknowledgements}

We are grateful to Dr Maria Grazia Volpe (Istituto di Scienze dell'Alimentazione, CNR, Avellino, Italy) for the preparation of PEFSO extract.

\section{References}

1. Guerriero E, Sorice A, Capone F, Napolitano V, Colonna G, Storti G, Castello G and Costantini S: Vitamin C effect on mitoxantrone-induced cytotoxicity in human breast cancer cell lines. PLoS One 9: e115287, 2014.

2. Iwamoto T: Clinical application of drug delivery systems in cancer chemotherapy: Review of the efficacy and side effects of approved drugs. Biol Pharm Bull 36: 715-718, 2013.

3. Smith L, Watson MB, O'Kane SL, Drew PJ, Lind MJ and Cawkwell L: The analysis of doxorubicin resistance in human breast cancer cells using antibody microarrays. Mol Cancer Ther 5: 2115-2120, 2006.

4. Rashid S, Ali N, Nafees S, Ahmad ST, Arjumand W, Hasan SK and Sultana S: Alleviation of doxorubicin-induced nephrotoxicity and hepatotoxicity by chrysin in Wistar rats. Toxicol Mech Methods 23: 337-345, 2013.

5. Rai G, Mishra S, Suman S and Shukla Y: Resveratrol improves the anticancer effects of doxorubicin in vitro and in vivo models: A mechanistic insight. Phytomedicine 23: 233-242, 2016.

6. Thakur JS, Chauhan CG, Diwana VK, Chauhan DC and Thakur A: Extravasational side effects of cytotoxic drugs: A preventable catastrophe. Indian J Plast Surg 41: 145-150, 2008.

7. Desai AG, Qazi GN, Ganju RK, El-Tamer M, Singh J, Saxena AK, Bedi YS, Taneja SC and Bhat HK: Medicinal plants and cancer chemoprevention. Curr Drug Metab 9: 581-591, 2008.

8. Vinod BS, Maliekal TT and Anto RJ: Phytochemicals as chemosensitizers: From molecular mechanism to clinical significance. Antioxid Redox Signal 18: 1307-1348, 2013.

9. Divakaran SA and Nai CKK: Amelioration of doxorubicin induced cardiotoxicity in tumor bearing mice by ferulic acid: A mechanistic study at cellular and biochemical level. Int J Tumor Ther 1: 6-13, 2012.

10. Chegaev K, Riganti C, Rolando B, Lazzarato L, Gazzano E, Guglielmo S, Ghigo D, Fruttero R and Gasco A: Doxorubicin-antioxidant co-drugs. Bioorg Med Chem Lett 23: 5307-5310, 2013.

11. Goyal A, Sharma V, Upadhyay N, Gill S and Sihag M: Flax and flaxseed oil: An ancient medicine \& modern functional food. J Food Sci Technol 51: 1633-1653, 2014.

12. Truan JS, Chen JM and Thompson LU: Comparative effects of sesame seed lignan and flaxseed lignan in reducing the growth of human breast tumors (MCF-7) at high levels of circulating estrogen in athymic mice. Nutr Cancer 64: 65-71, 2012.

13. Sorice A, Guerriero E, Volpe MG, Capone F, La Cara F, Ciliberto G, Colonna G and Costantini S: Differential response of two human breast cancer cell lines to the phenolic extract from flaxseed oil. Molecules 21: 319, 2016.

14. Capone F, Guerriero E, Sorice A, Colonna G, Storti G, Pagliuca J, Castello G and Costantini S: Synergistic antitumor effect of doxorubicin and tacrolimus (FK506) on hepatocellular carcinoma cell lines. Sci World J 2014: 450390, 2014.

15. Tirosh O, Sen CK, Roy S, Kobayashi MS and Packer L: Neuroprotective effects of alpha-lipoic acid and its positively charged amide analogue. Free Radic Biol Med 26: 1418-1426, 1999.

16. Chou TC: Theoretical basis, experimental design, and computerized simulation of synergism and antagonism in drug combination studies. Pharmacol Rev 58: 621-681, 2006.

17. Porichi O, Nikolaidou ME, Apostolaki A, Tserkezoglou A, Arnogiannaki N, Kassanos D, Margaritis L and Panotopoulou E: BCL-2, BAX and P53 expression profiles in endometrial carcinoma as studied by real-time PCR and immunohistochemistry. Anticancer Res 29: 3977-3982, 2009.

18. Stefanowicz-Hajduk J, Bartoszewski R, Bartoszewska S, Kochan K, Adamska A, Kosiński I and Ochocka JR: Pennogenyl saponins from Paris quadrifolia L. induce extrinsic and intrinsic pathway of apoptosis in human cervical cancer HeLa cells. PLoS One 10: e0135993, 2015.

19. Wang G, Zhang J, Liu L, Sharma S and Dong Q: Quercetin potentiates doxorubicin mediated antitumor effects against liver cancer through p53/Bcl-xl. PLoS One 7: e51764, 2012.

20. Maggio M, Artoni A, Lauretani F, Borghi L, Nouvenne A, Valenti $G$ and Ceda GP: The impact of omega-3 fatty acids on osteoporosis. Curr Pharm Des 15: 4157-4164, 2009.

21. Rodriguez-Leyva D, Dupasquier CM, McCullough R and Pierce GN: The cardiovascular effects of flaxseed and its omega-3 fatty acid, $\alpha$-linolenic acid. Can J Cardiol 26: 489-496, 2010 . 
22. Mason JK, Fu M, Chen J and Thompson LU: Flaxseed oil enhances the effectiveness of trastuzumab in reducing the growth of HER2-overexpressing human breast tumors (BT-474). J Nutr Biochem 26: 16-23, 2015 .

23. Sharifi S, Barar J, Hejazi MS and Samadi N: Doxorubicin changes $\mathrm{Bax} / \mathrm{Bcl}-\mathrm{xL}$ ratio, caspase- 8 and 9 in breast cancer cells. Adv Pharm Bull 5: 351-359, 2015.

24. Meiyanto E, Fitriasari A, Hermawan A, Junedi S and Susidarti RA: The improvement of doxorubicin activity on breast cancer cell lines by tangeretin through cell cycle modulation. Orient Pharm Exp Med 11: 183-190, 2011.

25. Cheng YY, Yang JS, Tsai SC, Liaw CC, Chung JG, Huang LJ, Lee KH, Lu CC, Chien HC, Tsuzuki M, et al: The newly synthesized 2-(3-hydroxy-5-methoxyphenyl)-6,7-methylenedioxyquinolin-4-one triggers cell apoptosis through induction of oxidative stress and upregulation of the p38 MAPK signaling pathway in HL-60 human leukemia cells. Oncol Rep 28: $1482-1490,2012$

26. Fulda $\mathrm{S}$ and Debatin KM: Extrinsic versus intrinsic apoptosis pathways in anticancer chemotherapy. Oncogene 25: 4798-4811, 2006.

27. Yang F, Chen H, Liu Y, Yin K, Wang Y, Li X, Wang G, Wang S, Tan X, Xu C, et al: Doxorubicin caused apoptosis of mesenchymal stem cells via p38, JNK and p53 pathway. Cell Physiol Biochem 32: 1072-1082, 2013.

28. Mi H, Lazareva-Ulitsky B, Loo R, Kejariwal A, Vandergriff J, Rabkin S, Guo N, Muruganujan A, Doremieux O, Campbell MJ, et al: The PANTHER database of protein families, subfamilies, functions and pathways. Nucleic Acids Res 33: D284-D288, 2005
29. Ortiz MA, Lopez-Hernandez FJ, Bayon Y, Pfahl M and Piedrafita FJ: Retinoid-related molecules induce cytochrome $c$ release and apoptosis through activation of c-Jun NH(2)-terminal kinase/p38 mitogen-activated protein kinases. Cancer Res 61: 8504-8512, 2001 .

30. Chuang SM, Wang IC and Yang JL: Roles of JNK, p38 and ERK mitogen-activated protein kinases in the growth inhibition and apoptosis induced by cadmium. Carcinogenesis 21: 1423-1432, 2000.

31. Ding H, Gabali AM, Jenson SD, Lim MS and Elenitoba-Johnson KS: P38 mitogen activated protein kinase expression and regulation by interleukin- 4 in human $B$ cell non-Hodgkin lymphomas. J Hematop 2: 195-204, 2009.

32. Parrish AB, Freel CD and Kornbluth S: Cellular mechanisms controlling caspase activation and function. Cold Spring Harb Perspect Biol 5: a008672, 2013.

33. Jin Z and El-Deiry WS: Overview of cell death signaling pathways. Cancer Biol Ther 4: 139-163, 2005.

34. Merhi F, Tang R, Piedfer M, Mathieu J, Bombarda I, Zaher M, Kolb JP, Billard C and Bauvois B: Hyperforin inhibits Akt1 kinase activity and promotes caspase-mediated apoptosis involving Bad and Noxa activation in human myeloid tumor cells. PLoS One 6: e25963, 2011. 